\title{
Performance of Various Metaheuristic Techniques for Economic Dispatch Problem with Valve Point Loading Effects and Multiple Fueling Options
}

\author{
Ijaz Ahmed, ${ }^{1,2}$ Ali Rauf Rao, ${ }^{1}$ Alaf Shah,, ${ }^{1}$ Engr Alamzeb, ${ }^{1}$ and Junaid Ali Khan ${ }^{1}$ \\ ${ }^{1}$ Faculty of Electrical Engineering, Hamdard University, Islamabad Campus, Islamabad 44000, Pakistan \\ ${ }^{2}$ Department of Electrical Engineering, Bahria University, Islamabad Campus, Islamabad 44000, Pakistan \\ Correspondence should be addressed to Ijaz Ahmed; ijazhere2000@gmail.com
}

Received 16 July 2014; Revised 5 November 2014; Accepted 6 November 2014; Published 27 November 2014

Academic Editor: Mamun B. Ibne Reaz

Copyright ( 92014 Ijaz Ahmed et al. This is an open access article distributed under the Creative Commons Attribution License, which permits unrestricted use, distribution, and reproduction in any medium, provided the original work is properly cited.

\begin{abstract}
The paper presents the metaheuristic approaches based on pattern search and simulated annealing hybridized with sequential quadratic programming, a powerful nonlinear adaptive technique for estimation of the finest combination of generated power in a given system at lowest operating cost while sustaining the operating condition of system efficiently. The fuel cost is minimized by satisfying the nonlinear operating conditions of thermal units mainly based on generation capacity constraints, generator ramp limit, power balance constraints, and valve point loading effect and by keeping in view the prohibited operating zones, respectively. About the optimization, a comparative study is made for pattern search (PS) and simulated annealing (SA), as a viable global search technique and sequential quadratic programming, an efficient local optimizer and their hybrid versions. The applicability, stability, and reliability of the designed approaches are validated through comprehensive statistical analysis based on Monte Carlo simulations.
\end{abstract}

\section{Introduction}

The performance and designing of electric power system serve two purposes; one is to maintain the economy and the other is to include reliability of the system [1]. Economic dispatch problem (EDP) is one of the significant optimization problems in industrial independent power system and acquires increasing importance as the operating cost and electric energy demand is increasing globally around the planet $[2,3]$. The procedure involves dividing the power demand of generating system for online thermal units in such manner that their operating cost is optimal while satisfying the power demand to the user and constraint adequately $[3,4]$. Since the operating cost of generating unit is inflated an optimal EDP can lead to prominent cost saving with reducing the atmospheric emission of thermal power plant [4]. In the energy crises age, a number of engineers and scientists are taking interest in the optimized solution of this industrial problem [5] by keeping in view the constraints like generator ramp limit, power balance, and valve point loading effect (VPLE) and prohibited operating zones [6,7]. However the facts of EDP with multiple fueling option (MFO) and MFO combined with VPLE conditions is not well addressed simultaneously, which are the motivations for taking this study with metaheuristic computing approach.

Traditional numerical models and programming strategies have been developed, like lambda iteration method, quadratic programming, Lagrangian relaxation algorithm, base point participation factor, gradient method linear programming, reduced gradient method, piecewise linear cost function, Newton method, and dynamic programming $[8$, 9], but all these EDP tools are flexible and applicable if the function between operating cost and fuel or heat are piecewise linear and increasing categorically [10]. Modern approaches use artificial neural network models which show dominance on classical techniques [11], while its parameters can overwrite during the learning in complex constraints based EDP problems. In order to overcome this issue the 
metaheuristic approaches based on tabu search, Hopfield, hill climbing, evolutionary computation, swarm intelligence, and ant/bee colony optimizations can outperform $[12,13]$.

In the recent development on optimization technique for EDP problems, multiperiod hydrothermal economic dispatch is employed with an efficient local search based on interior point method [14] that has an issue of getting stuck in the local minima. The solution of unit commitment problem is addressed by a hybrid scheme based on modified subgradient method and simulating annealing algorithm [15]; this combination of derivative based and derivative free method is good but with the constraints of high computational budget involved in its computations. The predictor corrector primal dual interior point algorithm is well presented in [16] in terms of computational cost for solving EDP problem; however the mean level of accuracy is not well matured. Parametric approach involved in parameter setting free harmony search and parallel adaptive algorithm schemes are established for economic dispatch and environmental power dispatch problems, respectively $[17,18]$. With all this recent development, this paper presents the comprehensive analysis in terms of minimum fuel cost, level of accuracy in the optimizer, statistical analysis on computational budget needed, and convergence rate as well, on complex dynamical version of EDP problem involving MFO and VPLE conditions. The presented work is compared with [19-21], in which EDP problem has been exploited by evolutionary strategy, standard particle swarm optimization (PSO), Gaussian PSO, and chaotic PSO, respectively.

The remaining of this paper is organized as follows: Section 2 describes the formulation of an EDP along with the constraints. The proposed methodology along with the procedural hybrid step is revealed in Section 3. Section 4 discusses the computational procedure and analyzes the results when applied to the scenarios of EDPs with 13,15, and 20 thermal units. The last section of the paper outlines the conclusions drawn from the simulation and results along with some directions for the future research.

\section{Economic Dispatch Problem Formulation}

The objective of EDP is to find the finest combination of generated power in a given system at lowest operating cost while sustaining the operating condition of system efficiently by satisfying the nonlinear operating conditions formulated as follow.

2.1. Fuel Cost Minimization. Assume we have a system where $\mathrm{n}$ thermal unit committed generating real power $P_{g i}$ and total fuel cost is $F_{c i}\left(P_{g i}\right)$; usually the fuel cost curve of committed units is assumed to be quadratic function of the real power output of generators. Consider

$$
\left.F_{T C}\right|_{\min }=\sum_{i=1}^{\mathrm{NG}} F_{c i}\left(P_{g i}\right)=\sum_{i=1}^{\mathrm{NG}}\left(A_{i}+B_{i} P_{g i}+C_{i} P_{g i}^{2}\right),
$$

where NG is the number of committed generators and $P_{g i}$ is real output power of generator in megawatt (MW) and can be

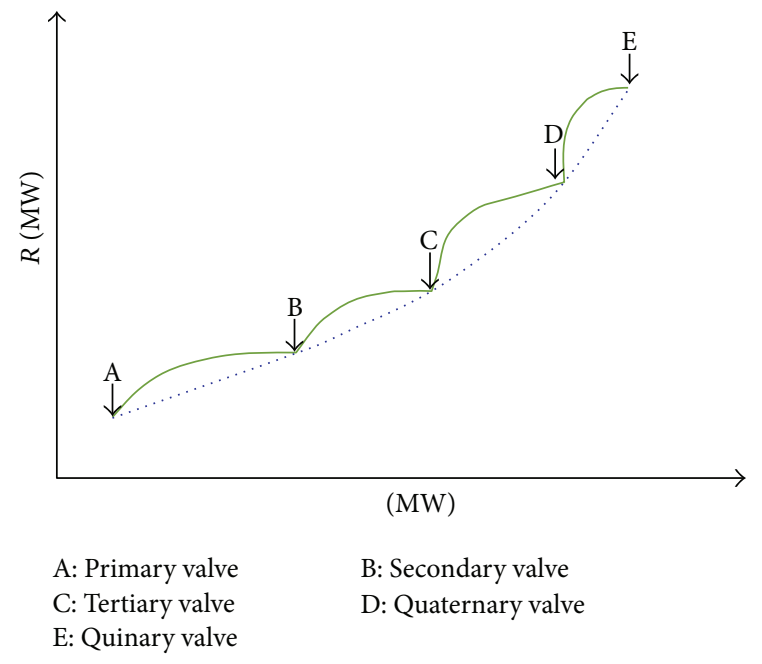

FIGURE 1: Cost function with primary, secondary, tertiary, quaternary, and quinary valves.

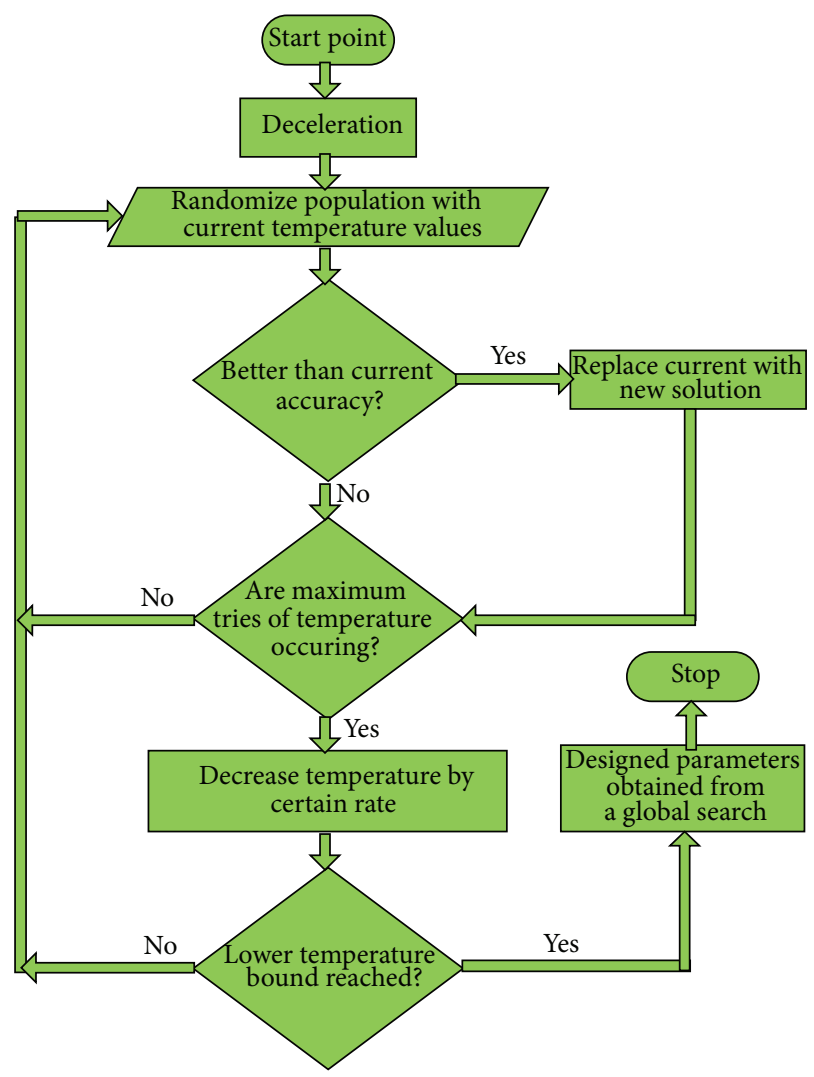

FIGURE 2: Flow diagram of metal of annealing algorithm.

represented as a real vector $P_{g i}=\left[p_{g 1}+p_{g 2}+p_{g 3}+\cdots+p_{g N G}\right]$, while $F_{T C}$ and $F_{C i}$ are the cost of all committed generators and cost of the $i$ th generator in terms of rupees/hour $(\mathrm{R} / \mathrm{h})$, respectively. $A_{i}, B_{i}$, and $C_{i}$ are the fuel-cost coefficient of $i$ th generator. 


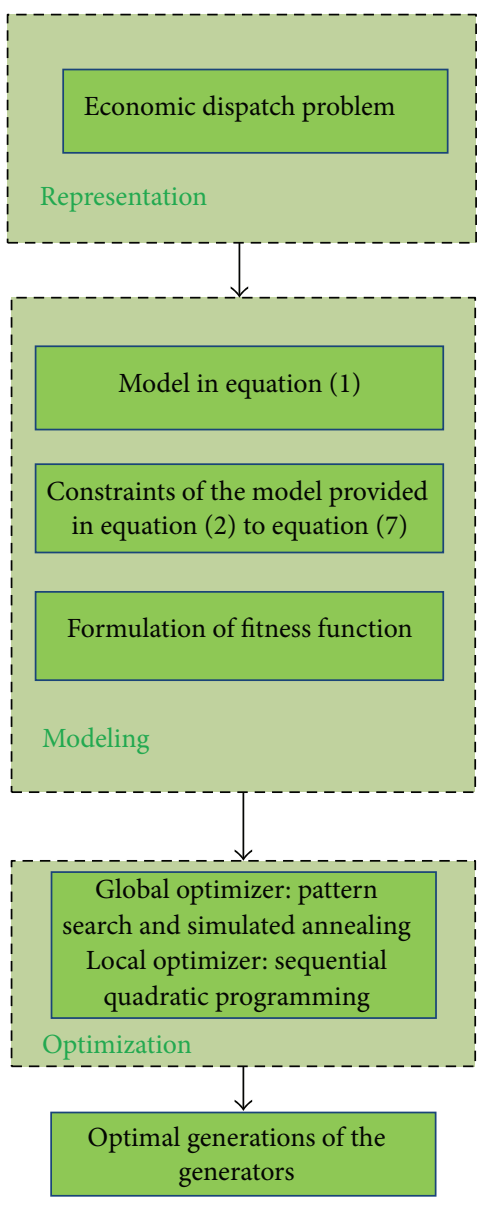

(a)

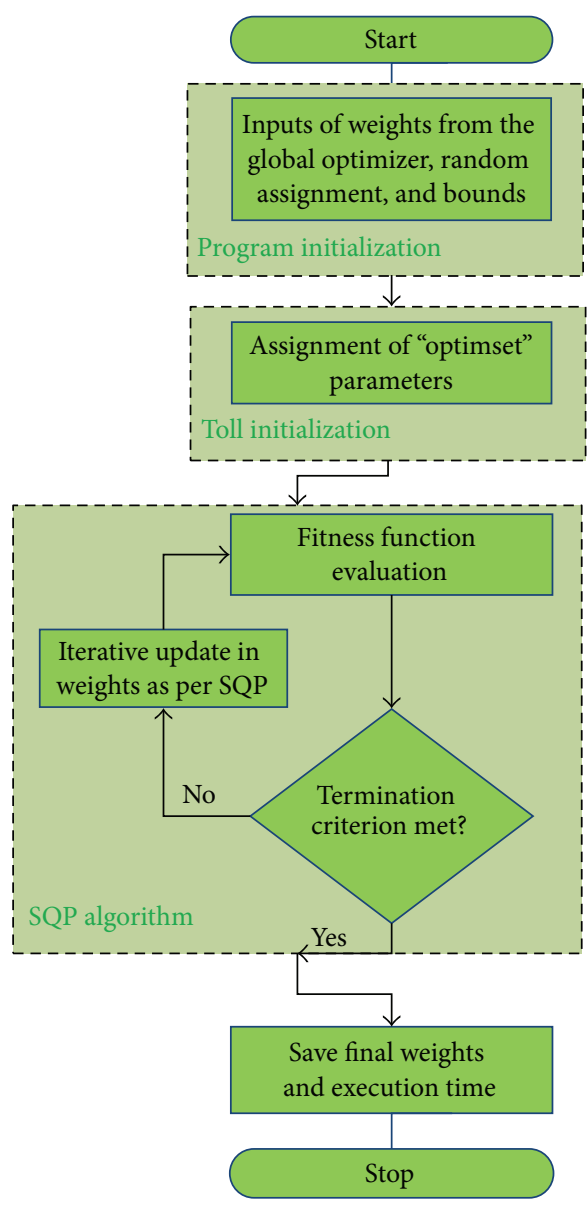

(b)

FIGURE 3: Process of proposed solution (a) overall procedure and (b) optimization of fitness function using hybrid computation.

\subsection{Inequality Constraint}

2.2.1. Generation Capacity Constraint. For the stable operation of each thermal unit the power generated, that is, $P_{g i}$, must be restricted by minimum power that must be generated and maximum power that a unit can deliver as follows:

$$
P_{g i}(\min ) \leq P_{g i} \leq P_{g i}(\max ), \quad i=1,2,3, \ldots, \text { NG }
$$

2.2.2. Generator Ramp Limit. With reference of [4] considering the ramp rate limit of generator, generation capacity constraint equation (2) can be modified as given below:

$$
\begin{array}{r}
\operatorname{Max}\left(P_{g i}(\min ), P_{g i}^{0}-\mathrm{DR}_{i}\right) \\
\leq P_{g i} \leq \operatorname{Min}\left(P_{g i}(\max ), P_{g i}^{0}+\mathrm{UR}_{i}\right) \\
\quad i=1,2,3, \ldots, \mathrm{NG},
\end{array}
$$

where $P_{g i}^{0}, \mathrm{UR}_{i}$, and $\mathrm{DR}_{i}$ are the previous operating point, upper ramp limit, and down ramp limit of the $i$ th generator, respectively.
2.2.3. Power Balance Constraint. Usually all the power plants are not working on same place they are spread geographically and also they must have some transmission power losses. The total power generated by committed thermal unit must be equal to the power demand of electric network plus the losses associated in power transmission [22]:

$$
\begin{gathered}
\sum_{i=1}^{N G} P_{g i}=P_{D}+P_{N L}, \\
P_{N L}=\sum_{i=1}^{N G} \sum_{j=1}^{N G} P_{i} B_{i j} P_{j}, \quad p_{i}=p_{g i}-p_{D}, \\
\beta_{i j}=\frac{R_{i j} \cos \left(\theta_{i}-\theta_{j}\right)}{\left|V_{i}\right|\left|V_{j}\right| \cos \theta_{i} \cos \theta_{j}} \\
\theta_{j}=\delta_{i}-\theta_{i}, \quad \theta_{i}=\tan \left(\frac{Q_{i}}{P_{i}}\right),
\end{gathered}
$$

where $P_{D}$ is the power demand, NG is number of generators, and $P_{N L}$ is the transmission power loss collective equation that is also known as coordination equation. The most 
TABle 1: Parameter setting/values using PS and SA.

\begin{tabular}{lccc}
\hline & & & SA \\
Parameters & Settings & Parameters & Setting \\
\hline Poll method & MADS positive basis 2N & Start point size & 15 \\
Poll order & Random & Population size & 300 \\
Mesh size & $(1,15)$ & Annealing function & Boltzmann \\
Expansion factor & 2.5 & Reannealing interval & 150 \\
Contraction factor & 0.7 & Temperature update & Exponential \\
Penalty factor & 150 & Initial temperature & 100 \\
Initial range & {$[0-1]$} & Bound & Problem specific \\
Maximum function Evaluations & 30000 & Maximum function evaluation & 45000 \\
Maximum iterations & 1500 & Maximum iterations & 1500 \\
Others & Default & Others & Default \\
\hline
\end{tabular}

TABle 2: Parameter setting/values using SQP method.

\begin{tabular}{lc}
\hline & Sequential quadratic programming \\
Parameters & Settings \\
\hline Start point & $\begin{array}{c}\text { Final weights of PS or SA } \\
\text { Specific lower and upper } \\
\text { limit of generators }\end{array}$ \\
Bounds & $10^{-12}$ \\
X-tolerance & $10^{-12}$ \\
Function tolerance & BFGS \\
Hessian & Objective and constraints \\
Scaling & 1000 \\
Maximum iterations & 1500 \\
Maximum function & Default \\
evaluations & Others
\end{tabular}

approximate and important method for transmission network losses as a function of generator power is $\beta$ coefficient method based on fact that, under normal operating conditions, the transmission loss is quadratic in the injected bus real power. Equation ( $4 \mathrm{~b}$ ) represents the general form of the loss formula using $\beta$ coefficient method. Here $N B, P_{i} P_{j}, Q_{i} Q_{j}$, and $Z_{i j}$ are the number of buses, the real power, reactive power of $i$ th and $j$ th buses, and elements of impedance matrix, respectively. However in this study $\beta$ coefficient method will be used so $\beta_{i j}$ are the loss constant of coefficient $\beta$.

2.3. Valve-Point Loading Effect (VPLE). For a power plant having NG committed units have multivalve opening sequence to meet the fluctuating loads demand. VPLE introduce ripples in the input-output characteristics of generating units resultantly making the heat curve rate nonconvex with increase in unit loading. Heat rate curve decreases between the opening points of two valves as shown in Figure 1 [23].

VPLE introduces ripples in the heat rate curve in multi valve opening turbine as shown in Figure 1, and for a perfect modeling of EDP a filter in the form of sinusoidal function must be added in objective function in this paper as given below:

$$
\begin{aligned}
F_{c i}\left(P_{g i}\right)= & A_{i}+B_{i} P_{g i}+C_{i} P_{g i}^{2} \\
& +\left|e_{i} \times \sin \left(f_{i} \times\left(P_{g i}^{\min }-P_{g i}\right)\right)\right|,
\end{aligned}
$$

where $e_{i}$ and $f_{i}$ are the coefficient of $i$ th unit that represents valve-point effect.

2.4. Prohibited Operating Zones. According to [3] practical generating units contain forbidden operating regions also known as "prohibited operating zones" due to machinery errors such as boiler pumps motors faults which ultimately leads input-output curve nonlinear and this can be modeled as follows:

$$
P_{g i} \in\left\{\begin{array}{l}
P_{g i}^{\min } \leq P_{g i} \leq P_{g i 1}^{N L} \\
P_{g i k-1}^{U} \leq P_{g i} \leq P_{g i k}^{N L} \\
P_{g i z I}^{U} \leq P_{g i} \leq P_{g i}^{\max },
\end{array}\right.
$$

where $P_{i}$ is the number of prohibited zones in the $i$ th generator curve, $k$ is the index of prohibited zone, and $P_{g i k}^{N L}$ and $P_{g i k}^{N L}$ are lower and upper limit of $k$ th prohibited zone of the $i$ th generator.

2.5. EDP with Multiple-Fueling Option (MFO). Another significant consideration involved in designing of EDP optimal cost is unit multiple fueling. A thermal unit has multiple cost rate curves because unit is fueled by different sources and needs to operate the unit on lower curve which result in producing hybrid cost function (HCF) and HCF contains information about unit operation or type of fuel used in generating operation. Practically multiple fuel sources are supplied to the thermal units and a single thermal unit can be represented by multiple quadratic cost functions; each cost function represents the type of fuel used and each unit must 
TABLE 3: Fitness evaluation values and their respective computational time for proposed schemes.

\begin{tabular}{lcccccccccc}
\hline \multirow{2}{*}{ Scenarios } & \multicolumn{2}{c}{ PS } & \multicolumn{2}{c}{ SA } & \multicolumn{2}{c}{ SQP } & \multicolumn{2}{c}{ PS-SQP } & \multicolumn{2}{c}{ SA-SQP } \\
& $F_{\text {val }}$ & $\tau$ & $F_{\text {val }}$ & $\tau$ & $F_{\text {val }}$ & $\tau$ & $F_{\text {val }}$ & $\tau$ & $F_{\text {val }}$ & $\tau$ \\
\hline Case 1 & $2.01 E-13$ & 0.29975 & $1.25 E-15$ & 26.9359 & $5.24 E-19$ & 0.09749 & $8.95 E-21$ & 0.22732 & $8.40 E-19$ & 0.05562 \\
Case 2 & $1.84 E-11$ & 1.68357 & $1.20 E-16$ & 29.8246 & $1.22 E-11$ & 2.98696 & $2.90 E-13$ & 1.10707 & $1.00 E-19$ & 0.24536 \\
Case 3 & $2.01 E-13$ & 0.29975 & $1.25 E-15$ & 26.9359 & $5.24 E-19$ & 0.09749 & $8.95 E-21$ & 0.22732 & $8.40 E-19$ & 0.05562 \\
\hline
\end{tabular}

TABLE 4: Reliability based on 100 independent runs for fifteen units system with $P_{D}=2630 \mathrm{MW}$.

\begin{tabular}{lccccc}
\hline Method & Min $_{\text {cost }}$ & Mean $_{\text {cost }}$ & STD $_{\text {cost }}$ & Max $_{\text {cost }}$ & Mean $_{\text {CPU }}$ time \\
\hline Evolutionary strategy [19] & 32568.54 & 32620.00 & - & 32710.00 & 0.48 \\
PSO [20] & 33858.00 & 33039.00 & - & 3331.000 & 26.59 \\
Genetic algorithm [20] & 33113.00 & 33228.00 & - & 3337.000 & 49.31 \\
Gaussian PSO [21] & 32508.12 & 35122.79 & 1918.62 & 38044.42 & 0.69 \\
Chaotic PSO [21] & 32775.68 & 35340.70 & 1942.84 & 38104.54 & \\
Proposed & & & & 36.001 .14 & \\
PS & 33154.10 & 34908.12 & 1712.34 & 36312.12 & 0.640587 \\
SA & 33124.24 & 35012.54 & 1612.35 & 35123.90 & 22.78067 \\
SQP & 32567.45 & 33467.14 & 1712.12 & 32568.28 & 0.418991 \\
PS-SQP & 32005.00 & 32145.23 & 1735.12 & 32412.02 & 1.059578 \\
SA-SQP & 32000.14 & 32112.12 & 1659.38 & 23.199661 \\
\hline
\end{tabular}

pin point the economic fuel to burn and fuel cost function formulation is as follows [23]:

$$
\begin{aligned}
& F_{c i}\left(P_{g i}\right) \\
& = \begin{cases}A_{i 1}+B_{i 1} P_{g i}+C_{i 1} P_{g i}^{2}, & \text { fuel } 1, P_{g i}^{\min } \leq P_{g i} \leq P_{g i 1} \\
A_{i 2}+B_{i 2} P_{g i}+C_{i 2} P_{g i}^{2}, & \text { fuel } 2, P_{g i} \leq P_{g i} \leq P_{g i 2} \\
\vdots & \vdots \\
A_{i k}+B_{i k} P_{g i}+C_{i k} P_{g i}^{2}, & \text { fuel } k, P_{g i k-1} \leq P_{g i} \leq P_{g i}^{\max },\end{cases}
\end{aligned}
$$

where $A_{i k}, B_{i k}$, and $C_{i k}$ are the cost coefficient of generator $i$ th using $k$ fuels.

2.6. EDP Considering Both VPLE and MFO. To obtain the realistic and transparent EDP solution in this paper both MFO and VPLE model are considered. Mathematical modeling for this consideration is combination of both cost functions given in (6) and (8). Consider

$$
F_{c i}\left(P_{g i}\right)= \begin{cases}A_{i 1}+B_{i 1} P_{g i}+C_{i 1} P_{g i}^{2}+\left|e_{i 1} \times \sin \left(f_{i 1} \times\left(P_{g i 1}^{\min }-P_{g i 1}\right)\right)\right|, & \text { fuel } 1, P_{g i}^{\min } \leq P_{g i} \leq P_{g i 1} \\ A_{i 2}+B_{i 2} P_{g i}+C_{i 2} P_{g i}^{2}+\left|e_{i 2} \times \sin \left(f_{i 2} \times\left(P_{g i 2}^{\min }-P_{g i 2}\right)\right)\right|, & \text { fuel } 2, P_{g i} \leq P_{g i} \leq P_{g i 2} \\ \vdots & \vdots \\ A_{i k}+B_{i k} P_{g i}+C_{i k} P_{g i}^{2}+\left|e_{i k} \times \sin \left(f_{i k} \times\left(P_{g i k}^{\min }-P_{g i k}\right)\right)\right|, & \text { fuel } k, P_{g i k-1} \leq P_{g i} \leq P_{g i}^{\max } .\end{cases}
$$

\section{Proposed Methodology}

The brief introduction of optimizers used for optimization of EDP is narrated along with the necessary detail of hybrid algorithm in the form of procedural steps.

3.1. Simulated Annealing. Simulated annealing is heuristic as well as probabilistic method proposed by Scott Kirkpatrick, C. Daniel Gelatt, and Mario P. Vecchi in 1983 for localizing better approximation to find global minimum of given cost function that may be surrounded with several local minima in a large search space [24]. SA is more efficient as compared with hill climbing and is a good choice for engineers and researchers to handle complex nonlinear systems especially problems like EDP due to its coding leniency to get guarantee finest optimal solutions. The method works on the principle of randomly initializing of finite solution space of specific size $N$ on the basis of scattered neighborhood points that mimic from the principle of thermal annealing of solids [25]. The decreasing of temperature at a specific rate in each iteration resembles the optimization of the merit function of EDP subject to varying the heat values as adaptive parameters of a specific problem. The candidate solution is updated based on the values of the fitness in terms of mean square error of the previous possible solution along with the index term; on this basis the iterative process matures towards 
TABLE 5: Reliability based on 100 independent runs for twenty units system with $P_{D}=2500 \mathrm{MW}$.

\begin{tabular}{|c|c|c|c|c|c|}
\hline Method & $\operatorname{Min}_{\text {cost }}$ & Mean $_{\text {cost }}$ & STD $_{\text {cost }}$ & Max $_{\text {cost }}$ & Mean $_{\mathrm{CPU}}$ time (s) \\
\hline Lambda iteration method [38] & 62456.6391 & - & - & - & 0.033 \\
\hline Hopfield neural networks [38] & 62456.6341 & - & - & - & 0.006 \\
\hline Gaussian PSO [21] & 60526.15 & 61123.36 & 330.89 & 62757.19 & 0.50 \\
\hline Chaotic PSO [21] & 60803.51 & 61223.95 & 507.56 & 62982.19 & 0.37 \\
\hline \multicolumn{6}{|l|}{ Proposed } \\
\hline PS & 59829.15 & 61256.21 & 1342.56 & 63125.56 & 1.054175 \\
\hline SA & 59803.45 & 61002.34 & 1423.89 & 62340.16 & 32.31666 \\
\hline SQP & 57231.43 & 59012.45 & 1712.35 & 60123.23 & 0.294808 \\
\hline PS-SQP & 58123.98 & 58563.65 & 976.140 & 58901.26 & 1.348983 \\
\hline SA-SQP & 57120.21 & 58011.78 & 658.341 & 58764.38 & 33.643791 \\
\hline
\end{tabular}

TABLE 6: Data for thirteen thermal units of generating unit capacity and coefficient [37].

\begin{tabular}{lccccc}
\hline Units & $\begin{array}{c}P_{i}^{\min } \\
(\mathrm{MW})\end{array}$ & $\begin{array}{c}P_{i}^{\max } \\
(\mathrm{MW})\end{array}$ & $\begin{array}{c}a \\
\left(\$ / \mathrm{MW}^{2}\right)\end{array}$ & $\begin{array}{c}b \\
\left(\$ / \mathrm{MW}^{2}\right)\end{array}$ & $\begin{array}{c}c \\
\left(\$ / \mathrm{MW}^{2}\right)\end{array}$ \\
\hline 1 & 0 & 680 & 0.00028 & 8.10 & 550 \\
2 & 0 & 360 & 0.00056 & 8.10 & 309 \\
3 & 0 & 360 & 0.00056 & 8.10 & 307 \\
4 & 60 & 180 & 0.00028 & 7.74 & 240 \\
5 & 60 & 180 & 0.00324 & 7.74 & 240 \\
6 & 60 & 180 & 0.00324 & 7.74 & 240 \\
7 & 60 & 180 & 0.00324 & 7.74 & 240 \\
8 & 60 & 180 & 0.00324 & 7.74 & 240 \\
9 & 60 & 180 & 0.00324 & 7.74 & 240 \\
10 & 40 & 120 & 0.00284 & 8.60 & 126 \\
11 & 40 & 120 & 0.00284 & 8.60 & 126 \\
12 & 55 & 120 & 0.00284 & 8.60 & 126 \\
13 & 55 & 120 & 0.00284 & 8.60 & 126 \\
\hline
\end{tabular}

the region of convergence of the fitness function. SA belongs to the family of global search techniques so it has various start points in the start of the algorithm to avoid local minimum. Usually the number of iterations of SA is very large while the computational budget is quite reasonable for most of the test bench problem. Although, it has been applied in various areas of engineering, some key applications are found in crystallographic refinement, neural computing [26], optimal reconfiguration of distribution networks, wireless communication, phase balancing, van der pol oscillators involved in mechanical engineering [27], and combinatorial optimization problem [28]. The generic flow diagram used for the simulated annealing algorithm is provided in Figure 2.

3.2. Pattern Search. Pattern search (PS) is numerical optimization method proposed by Hooke and Jeeves [29] for statistical problems and main advantages of PS are that it is efficient and computational structure is simple to understand and also it does not required gradient of cost function so it can be used in functions that are not constant or not differentiable and method is also known as direct-search, derivative-free approaches. Pattern search algorithm searches for a sequence of points to reach an optimal solution by initializing a set of points called mesh. The mesh formation procedure is involved by adding a pattern vector to the initial point and if PS acquires a point in mesh that refines the objective function then the new point becomes the current point at the next step of the algorithm [30].

Usually the practical nonlinear optimization problem of power system lies outside the reach of the standard optimization techniques. The practical EDP problems can be optimized by PS which is computationally efficient and easy to implement as compared to other heuristic techniques [31]. PS initializes by computing sequence of point that may or may not be reached optimally and established a set point called mesh around these given points. This current point could be a starting point provided by user or extracted from previous step of algorithm. Current points are then added to a pattern vector which is scalar multiple of a set of vectors for mesh formation. If an objective function is improved by point in mesh then the new point becomes the current point at the next iteration [32].

Pattern search is efficient optimization techniques applied in areas of parallel computing, pattern character recognition, graphic user interference thermal control systems, nonlinear and optimal control theory [33], and so forth.

3.3. Sequential Quadratic Programming. The SQP algorithm is one of the nonlinear programming techniques used extensively by the research community for constraints optimization problems. The strength of the scheme is well-established in terms of efficiency, accuracy, and percentage of successful solutions over a large number of test problems. Thum and Schilling [34] have provided excellently a brief introduction and description of SQP algorithms. As the physics of SQP is based on local search techniques so it is more probable to get stuck in the local minimum so some of the iterations during Monte Carlo simulation can show divergence or least accuracy. However the rate of convergence and level of accuracy for successful runs is dramatically high for SQP algorithms. The detailed history, mathematical formulation, importance, and application related with SQP methods can be seen in $[35,36]$. The SQP methods have been applied on broad disciplines of engineering and applied science from 
TABLE 7: (a) Data for fifteen thermal units of generating unit capacity and coefficient [19-21]. (b) Data for fifteen thermal units of generating units of ramp-rate limits and prohibited zones of generating units.

(a)

\begin{tabular}{lccccc}
\hline Units & $P_{i}^{\min }(\mathrm{MW})$ & $P_{i}^{\max }(\mathrm{MW})$ & $a\left(\$ / \mathrm{MW}^{2}\right)$ & $b\left(\$ / \mathrm{MW}^{2}\right)$ & $c\left(\$ / \mathrm{MW}^{2}\right)$ \\
\hline 1 & 150 & 455 & 0.000299 & 10.1 & 671 \\
2 & 150 & 455 & 0.000183 & 10.2 & 574 \\
3 & 20 & 130 & 0.001126 & 8.8 & 374 \\
4 & 20 & 130 & 0.001126 & 10.4 & 374 \\
5 & 150 & 470 & 0.000205 & 10.1 & 461 \\
6 & 135 & 460 & 0.000301 & 9.8 & 630 \\
7 & 135 & 465 & 0.000364 & 11.2 & 548 \\
8 & 60 & 300 & 0.000338 & 11.2 & 227 \\
9 & 25 & 162 & 0.000807 & 10.7 & 173 \\
10 & 25 & 160 & 0.001203 & 10.2 & 175 \\
11 & 20 & 80 & 0.003586 & 9.9 & 186 \\
12 & 20 & 80 & 0.005513 & 13.1 & 230 \\
13 & 25 & 85 & 0.000371 & 12.1 & 30 \\
14 & 15 & 55 & 0.001929 & 12.4 & 325 \\
15 & 15 & 55 & 0.004447 & & 323 \\
\hline
\end{tabular}

(b)

\begin{tabular}{|c|c|c|c|c|c|c|}
\hline \multirow{2}{*}{ Units } & \multirow{2}{*}{$P_{i}^{0}(\mathrm{MW})$} & \multirow{2}{*}{$\mathrm{UR}_{i}(\mathrm{MW} / \mathrm{h})$} & \multirow{2}{*}{$\mathrm{DR}_{i}(\mathrm{MW} / \mathrm{h})$} & \multicolumn{3}{|c|}{ Prohibited zones } \\
\hline & & & & Zone 1 & Zone 2 & Zone 3 \\
\hline 1 & 400 & 80 & 120 & & & \\
\hline 2 & 300 & 80 & 120 & [185 255] & [305 335] & {$\left[\begin{array}{lll}420 & 450\end{array}\right]$} \\
\hline 3 & 105 & 130 & 130 & & & \\
\hline 4 & 100 & 130 & 130 & & & \\
\hline 5 & 90 & 80 & 120 & {$\left[\begin{array}{ll}180 & 200\end{array}\right]$} & [305 335] & {$\left[\begin{array}{lll}390 & 420\end{array}\right]$} \\
\hline 6 & 400 & 80 & 120 & [230 255] & [365 395] & {$\left[\begin{array}{lll}430 & 455\end{array}\right]$} \\
\hline 7 & 350 & 80 & 120 & & & \\
\hline 8 & 95 & 65 & 100 & & & \\
\hline 9 & 105 & 60 & 100 & & & \\
\hline 10 & 110 & 60 & 100 & & & \\
\hline 11 & 60 & 80 & 80 & & & \\
\hline 12 & 40 & 80 & 80 & [30 40] & {$[5565]$} & \\
\hline 13 & 30 & 80 & 80 & & & \\
\hline 14 & 20 & 55 & 55 & & & \\
\hline 15 & 20 & 55 & 55 & & & \\
\hline
\end{tabular}

the day of their birth to today; for instance, few recently published articles addressing SQP technique are referred to for interested readers in $[37,38]$.

Now the intention is to formulate the overall procedure and the optimization of the unknown designed parameters of the units generation along with the fuel cost; the process of the proposed scheme is provided in Figure 3. The modeling is performed based on relations provided in (1) and relations of constraints, respectively. The optimization is performed using global versions of simulated annealing and pattern search hybrid with sequential quadratic programming, an efficient local search technique. In Figure 3(b) the designed parameters obtained from global optimizer are provided as a start point to SQP for further tuning of the design. The algorithm used for the optimization of EDP in the form of the logical steps based on hybrid computational technique is as follows.

Step 1 (population generation). A random and well scattered population of size $M$ is generated with agents of length exactly equal to the number of power generating units $N$.

In general the $i$ th agent of the candidate solution is represented by $\mathbf{c}_{i}=\left[c_{i 1}, c_{i 2}, \ldots, c_{i N}\right], c_{i j} \in \Re: L_{b} \leq c_{i j} \leq U_{b} \forall i=1$, $2,3, \ldots, N, j=1,2,3, \ldots, M$, where $L_{b}$ and $U_{b}$ are the lower and upper possible limits to generate power.

Step 2 (initialization). Initialize and assign the general and specific values for parameter setting involved in pattern search and simulated annealing algorithm as provided in Table 1 . 
TABLE 8: Data for twenty thermal generating units capacity and coefficients (20 units) [19-21].

\begin{tabular}{lccccc}
\hline Units & $\begin{array}{c}P_{i}^{\min } \\
(\mathrm{MW})\end{array}$ & $\begin{array}{c}P_{i}^{\max } \\
(\mathrm{MW})\end{array}$ & $\begin{array}{c}a \\
\left(\$ / \mathrm{MW}^{2}\right)\end{array}$ & $\begin{array}{c}b \\
\left(\$ / \mathrm{MW}^{2}\right)\end{array}$ & $\begin{array}{c}c \\
\left(\$ / \mathrm{MW}^{2}\right)\end{array}$ \\
\hline 1 & 150 & 600 & 0.00068 & 18.19 & 1000 \\
2 & 50 & 200 & 0.00071 & 19.26 & 970 \\
3 & 50 & 200 & 0.00650 & 19.80 & 600 \\
4 & 50 & 200 & 0.00500 & 19.10 & 700 \\
5 & 50 & 160 & 0.00738 & 18.10 & 420 \\
6 & 20 & 100 & 0.00612 & 19.26 & 360 \\
7 & 25 & 125 & 0.00790 & 17.14 & 490 \\
8 & 50 & 150 & 0.00813 & 18.92 & 660 \\
9 & 50 & 200 & 0.00522 & 18.27 & 765 \\
10 & 30 & 150 & 0.00573 & 18.92 & 770 \\
11 & 100 & 300 & 0.00480 & 16.69 & 800 \\
12 & 150 & 500 & 0.00310 & 16.76 & 970 \\
13 & 40 & 160 & 0.00850 & 17.36 & 900 \\
14 & 20 & 130 & 0.00511 & 18.70 & 700 \\
15 & 25 & 185 & 0.00398 & 18.70 & 450 \\
16 & 20 & 80 & 0.07120 & 14.26 & 370 \\
17 & 30 & 85 & 0.00890 & 19.14 & 480 \\
18 & 30 & 120 & 0.00713 & 18.92 & 680 \\
19 & 40 & 120 & 0.00622 & 18.47 & 700 \\
20 & 30 & 100 & 0.00773 & 19.79 & 850 \\
\hline & & & & &
\end{tabular}

Step 3 (fitness evaluation). Find the fitness of each agent using the fitness evaluation function $\xi$ as given below in the form of mean square error (MSE):

$$
\xi=\left(\frac{\sum_{j=1}^{N}\left(\left|C_{\mathrm{op}}^{j}-\widehat{C}_{\mathrm{op}}^{j}\right|^{2}\right)}{N}\right),
$$

where $\widehat{C}_{\mathrm{op}}^{i}$ is the approximated operating cost for $j$ th generator.

Step 4 (termination criteria). The algorithm is terminated on the basis of the following criteria.

(a) The predefined fitness value is achieved.

(b) The total number of generations is executed.

(c) Predefined value of the function tolerance is achieved.

(d) The desired function evaluations are performed.

If any of the above criteria are met then go to Step 6 otherwise simulate Step 5.

Step 5 (reproduction). New population is generated using the operations evolved in PS and SA as per the setting given in Table 1 and then go to Step 3.

Step 6 (refinement via SQP method). One of the best results obtained by PS and SA is provided to SP as a start point for further refinement, and the parameter setting and values used for SQP algorithm are provided in Table 2.
Step 7 (storage). Store the global best of this cycle and repeat the procedure from Steps 3 to 7, to get sufficient numbers of independent runs to have sufficient data for performing analysis.

Step 8 (statistical analysis). The statistical analysis is made on the basis of four fundamental performance modes like best, worst, mean, and standard deviation and skewness.

\section{Simulation and Results}

In this section, a scenario based simulation is performed based on three case studies composed of thirteen, fifteen, and twenty generating units, respectively, for EDP problem in which the objective functions were ramp rate limit, prohibited operating zones in the power system operation; MFO and VPLE and transmission losses are also employed to demonstrate efficiency of proposed schemes using MATLAB built in subroutines of the optimizers like PS, SA, SQP, PSSQP, and SA-SQP, respectively, whose parameters setting and values are provided in Tables 1 and 2. The reference data sheet for thirteen thermal units [37] with load demand of $3080 \mathrm{MW}$ is provided in Appendix A. The data set used for fifteen thermal units [19-21] along with generating unit capacity, coefficients, ramp rate limits, and prohibited zones is provided in Appendix B while the load demand expected to be determined is $2630 \mathrm{MW}$. However, the data sheet for twenty thermal units of generating unit capacity and coefficients [38] are provided in Appendix C, where system supplies a total load demand of $2500 \mathrm{MW}$.

One of the best designed parameters is in fact the power generating values of generators and is presented in Figure 4 at semilog scale in order to widen the small effects in the power values. The lowest cost of the power generation can be obtained by taking the generating values from the hybrid schemes based on PS-SQP and SA-SQP, respectively, from Figures 4(a), 4(b), and 4(c) in fitness evaluation function provided in (10) along with the subsequent relations given in (1) and expressions of EDP constraints. The $x$-axis in Figure 4 defines the number of thermal units taken in the study and $y$ axis demonstrates the solvers PS, SA, SQP, PS-SQP, and SASQP, respectively, while $z$-axis provides the optimized values of the power for thermal units.

The optimized final values along with computational time for PS, SA, SQP, PS-SQP, and SA-SQP are provided in Table 3. The time analysis carried out for this paper is on Intel(R) Core (TM) i5-3210M CPU @ 2.50 GHz, 2.5 GHz, 4.0 GB of RAM, and running with MATLAB version 2012a. It is quite evident from the table that level of accuracy is dominated in case of hybrid schemes as compared to global search and local search techniques separately. However the computational budget of hybrid approach is a little bit more as compared to other presented schemes but this effect can be over shed due to the high accuracy attained by hybrid schemes. The local search scheme seems to be outperformed in terms of both accuracy and computational cost; however one cannot rely on one of these best values of SQP as the local search techniques are more probable to get stuck in the local optima due to bad initial guess. Another observation is that as the number of 
TABLE 9: Reliability based on 100 independent runs for fifteen units system with $P_{D}=3080 \mathrm{MW}$ [37].

\begin{tabular}{|c|c|c|c|c|c|}
\hline Method & Minimum cost & Mean cost & STD of cost & Maximum cost & Mean CPU time \\
\hline \multicolumn{6}{|l|}{ Proposed } \\
\hline PS & 17524.78 & 17659.34 & 1543.72 & 17860.12 & 0.394373 \\
\hline SA & 17598.19 & 17623.78 & 1872.83 & 17765.23 & 14.15776 \\
\hline SQP & 17653.11 & 17723.14 & 1712.34 & 189023.1 & 0.148936 \\
\hline PS-SQP & 17201.98 & 17543.76 & 1612.35 & 17678.91 & 0.543309 \\
\hline SA-SQP & 17491.17 & 17502.12 & 1709.12 & 17233.24 & 14.306696 \\
\hline
\end{tabular}

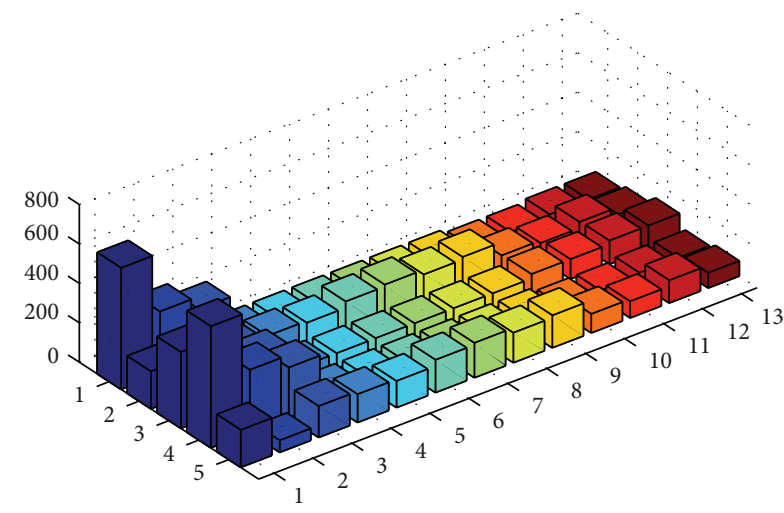

(a)

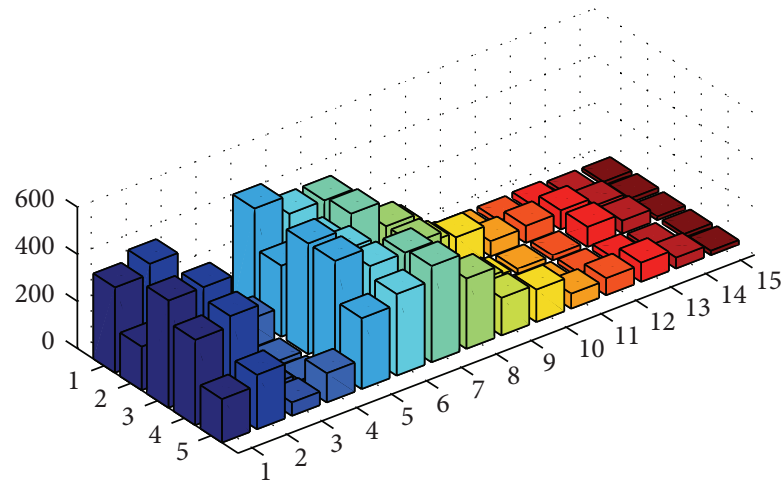

(b)

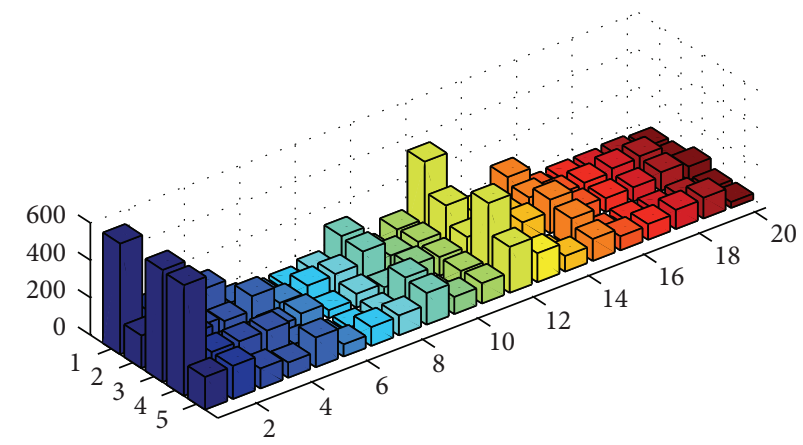

(c)

FIGURE 4: Analysis of the optimizers for 100 independent runs for (a) thirteen generating units, (b) fifteen generating units, and (c) twenty generating units.

generating units increases the level of accuracy reduces while computational time increases due to increase in the EDP complexity.

The reliability of the stochastic algorithms is being tested by a comprehensive statistical analysis in terms of minimum of cost $\left(\mathrm{Min}_{\text {cost }}\right)$, maximum value of cost $\left(\operatorname{Max}_{\text {cost }}\right)$, mean cost value $\left(\right.$ Mean $\left._{\text {cost }}\right)$, and standard deviation of the cost $\left(\mathrm{STD}_{\text {cost }}\right)$. The analysis is not only performed on cost value but also performed on computational time needed for the optimization in term of mean CPU $\left(\right.$ Mean $\left._{\mathrm{CPU}}\right)$ time. The results of the Monte Carlo simulations for stochastic solvers are narrated in Tables 4 and 5 for fifteen and twenty units, respectively, while for thirteen units the same analysis is provided in Appendix D.

It is quite evident from Tables 4 and 5 that the results of the proposed schemes are in good agreement in terms of reliability, effectiveness, economical cost, and computational budget required in terms of time complexity; however the hybrid approaches PS-SQP and SA-SQP show more supremacy than that of PS, SA, and SQP. The computational budget of hybrid schemes seems to computationally heavy of order of just few seconds; however this aspect seems to be minimal at the cost of level of accuracy achieved.

As the proposed scheme is based on heuristic computation one of the best results cannot define the effectiveness and reliability of the schemes; therefore in order to see the effectiveness of given stochastic methods the results are obtained for hundred independent runs rather on a single successful run. The fitness given in (10) obtained on all independent iterations is plotted in Figure 5 for three variations of generating units.

The percentage of convergence is also calculated by fixing a certain level of the threshold $\left(\xi \leq 10^{-10}\right)$ in the level of fitness obtained by all proposed algorithms and the results 


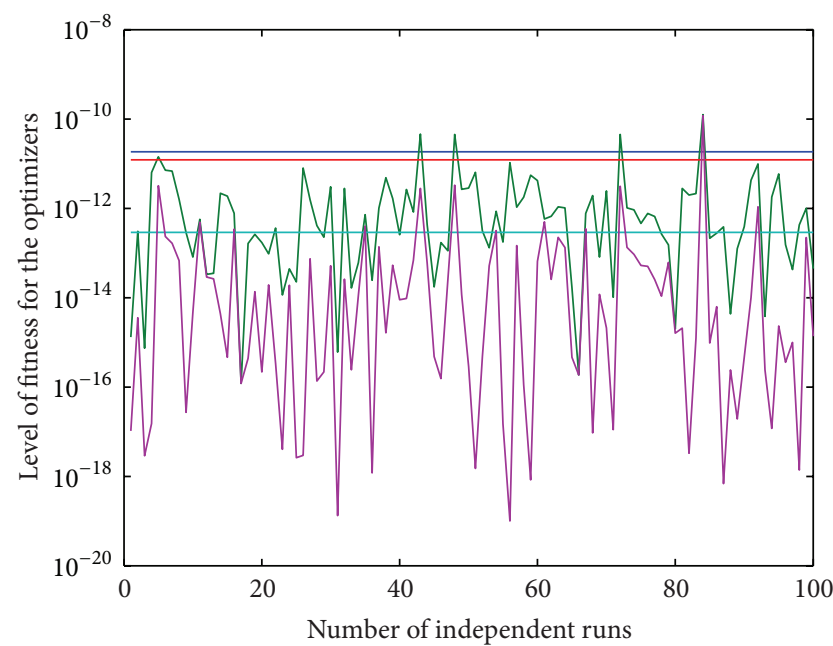

(a)

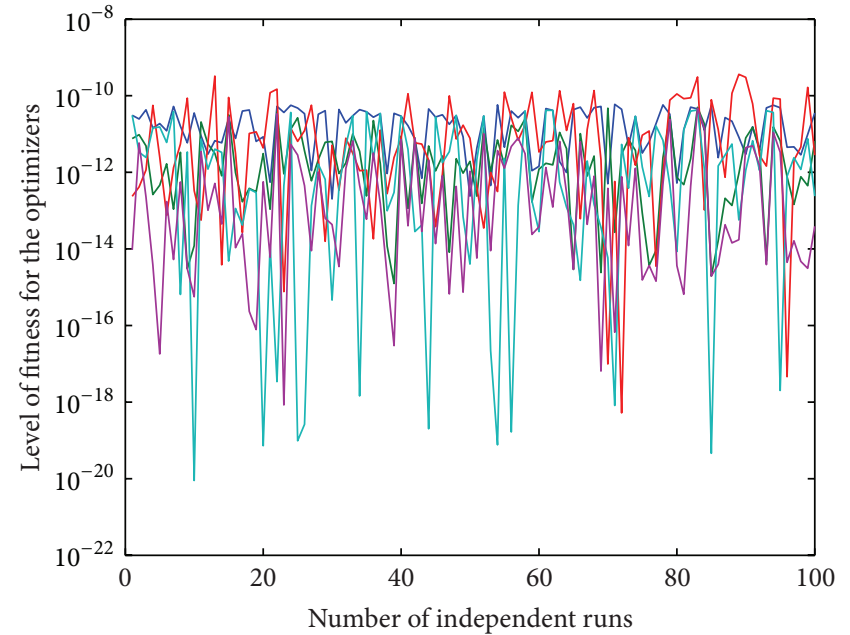

(b)

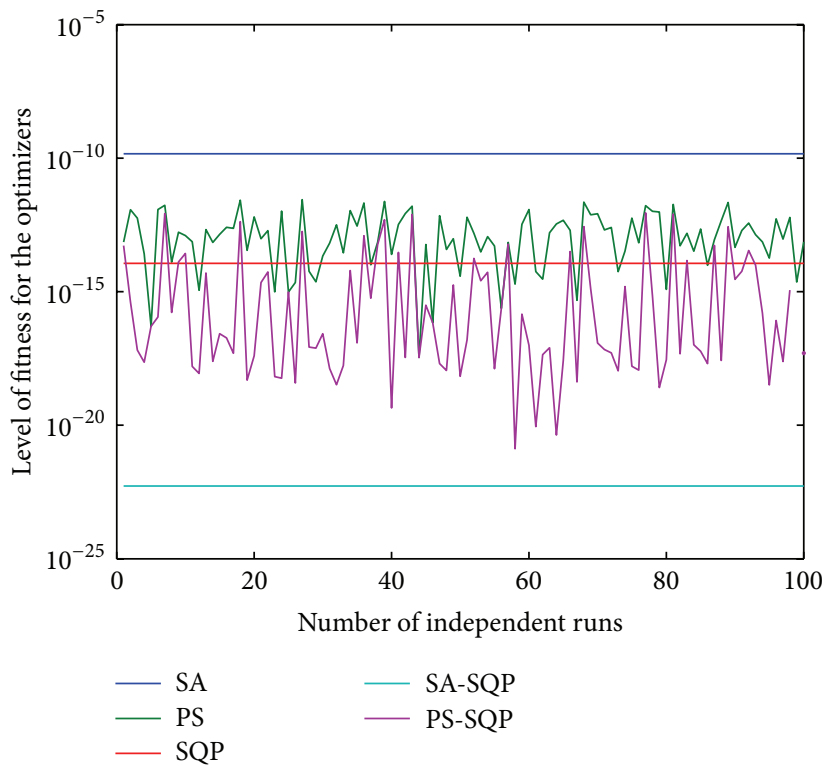

(c)

FIGURE 5: Process of proposed solution (a) overall procedure and (b) optimization of fitness function using hybrid computation.

are plotted in Figure 6 at four hundred independent runs of the given algorithms. It is observed from Figure 6(a) that all the optimizers provide $100 \%$ convergence for EDP with thirteen thermal units while for the case of fifteen units the convergence for SA is $97 \%$ and $100 \%$ for rest of the algorithms. Scenario three that is relatively stiff does not show convergence for PS on a threshold of $\left(\xi \leq 10^{-10}\right)$, while the other optimizers like SA, SQP, PS-SQP, and SA-SQP are 100\% convergent as evident from Figure 6(c).

\section{Conclusions}

On the basis of the results obtained by various optimizers in the last sections, the following conclusion can be drawn.

The proposed scheme based on SA-SQP and PS-SQP hybrid algorithms can provide reliable, effective, and well optimized cost of EDP for the scenarios of thirteen, fifteen, and twenty thermal units for a demand of $3080 \mathrm{M}, 2630 \mathrm{MW}$, and $2500 \mathrm{MW}$, respectively.

The comparative study between the solvers is carried out in terms of absolute cost, computational complexity, and fitness value achieved by the PS, SA, SQP, PS-SP, and SA-SP algorithms. The hybrid algorithms are found to be better than that of global and local search techniques applied independently for all variants of ESPs.

The results of statistical analysis determined for 100 independent runs of the design approaches show that the values in terms of mean, standard deviation, and minimum of the total cost error associated with PS-SQP and SASQP are considerably more dominated than SA, PS, and SQP techniques. The value of the mean execution time for finding the optimal weight with proposed schemes shows 


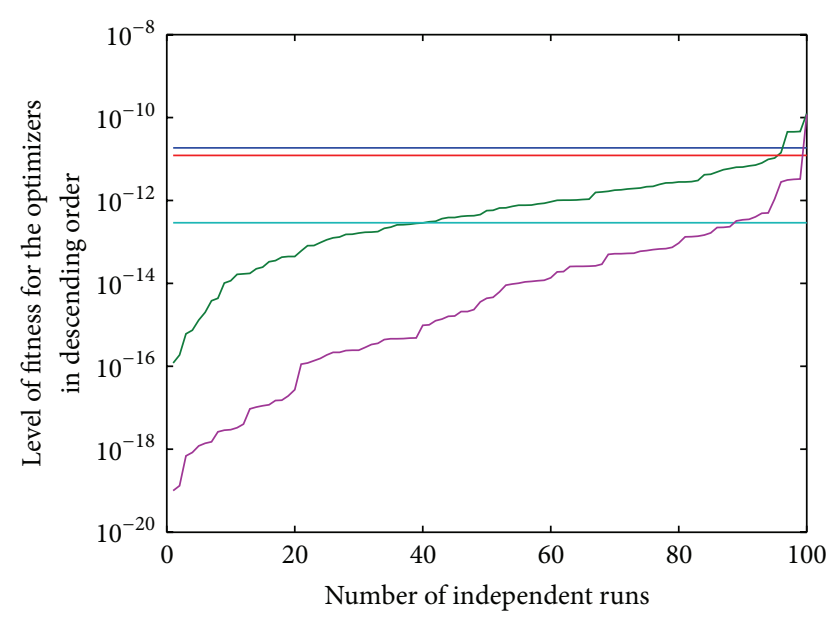

(a)

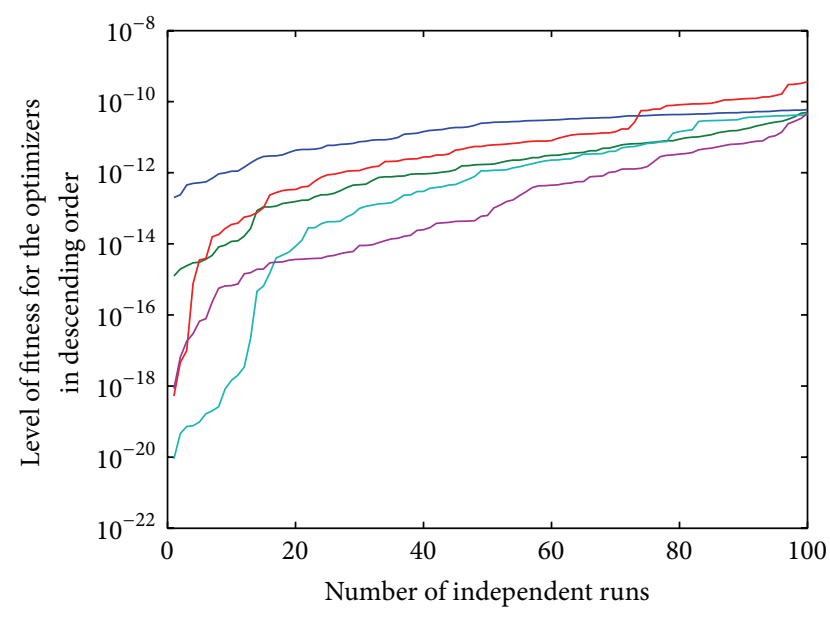

(b)

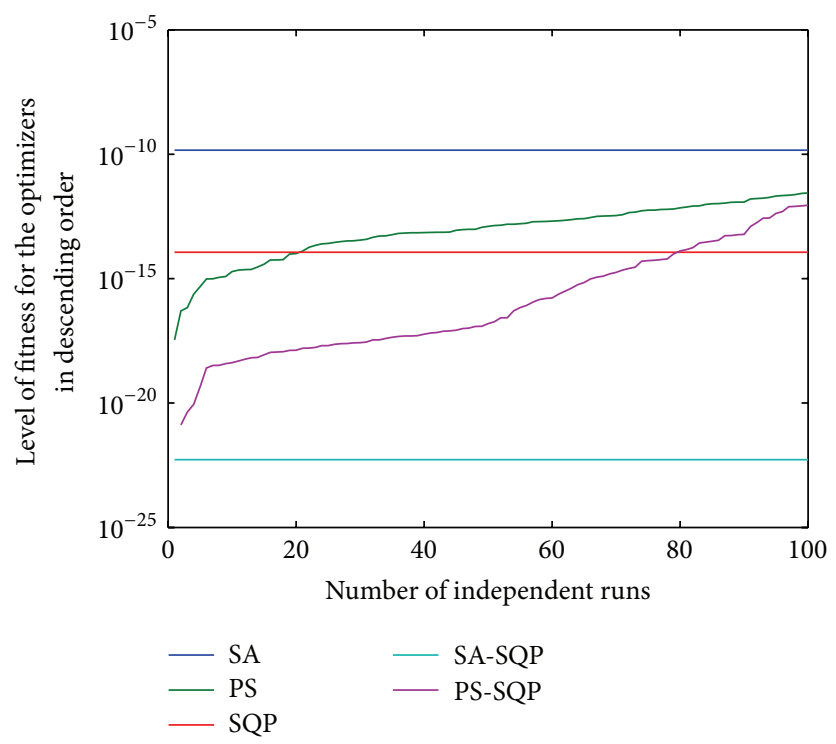

(c)

Figure 6: Process of proposed solution (a) overall procedure and (b) optimization of fitness function using hybrid computation.

that the hybrid approaches take relatively more time than the other three techniques but this exception can be overshadow due to its utmost accuracy and consistently convergent performance.

At a fitness threshold of $\varepsilon \leq 10^{-10}$, the algorithms based on hybrid schemes on average provide $100 \%$ convergence while the convergences of SA and PS are observed to be in a good range of acceptance, although in same situations PS remains consistent due to the same creation of mesh in the polling and searching domain.

The main advantages of given schemes lie in their ease in implementation, simplicity in the concept, minimal computational complexity, less hardware implementation burden, and suitability for real-time applications. In future, one may look for biological inspired computational method to analyze synthesis and optimize the other dynamical constraint based EDPs problems.

\section{Appendices}

\section{A. Generating Units Capacity and Coefficients (13 Units)}

See Table 6

\section{B. (a) Generating Units Capacity and Coefficients (15 Units) (b) Ramp-Rate Limits and Prohibited Zones (15 Units)}

See Table 7.

\section{Generating Units Capacity and Coefficients (20 Units)}

See Table 8. 


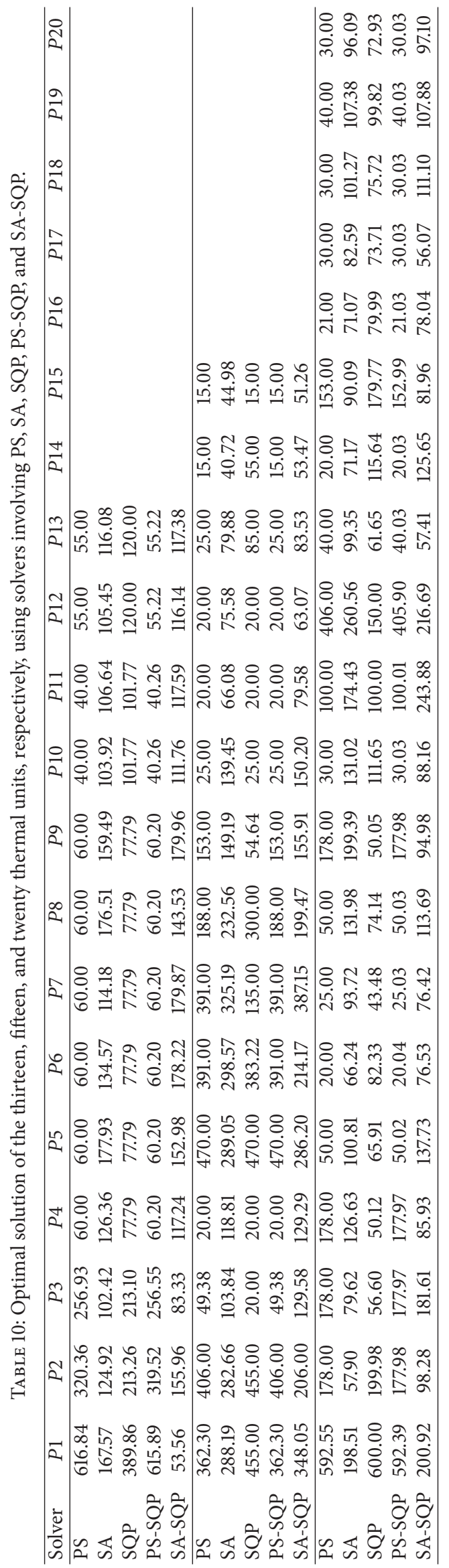




\section{Reliability Comparison Based upon 100 Independent Runs for 15 Units with $P_{D}=3080 \mathbf{M W}$}

See Table 9.

\section{E. Optimal Solution for 13, 15, and 20 Units Using Solvers Involving PS, SA, SQP, PS- SQP, and SA-SQP}

See Table 10.

\section{Conflict of Interests}

The authors declare that there is no conflict of interests regarding the publication of this paper.

\section{References}

[1] P.-H. Chen and H.-C. Chang, "Large-scale economic dispatch by genetic algorithm," IEEE Transactions on Power Systems, vol. 10, no. 4, pp. 1919-1926, 1995.

[2] R. K. Swaina, N. C. Sahub, and P. K. Hotac, "Gravitational search algorithm for optimal economic dispatch," Procedia Technology, vol. 6, pp. 411-419, 2012, Proceedings of the 2nd International Conference on Communication, Computing \& Security (ICCCS '12).

[3] R. Poli, "Analysis of the publications on the applications of particle swarm optimisation," Journal of Artificial Evolution and Applications, vol. 2008, Article ID 685175, 10 pages, 2008.

[4] Z.-L. Gaing, "Particle swarm optimization to solving the economic dispatch considering the generator constraints," IEEE Transactions on Power Systems, vol. 18, no. 3, pp. 1187-1195, 2003.

[5] D. He, F. Wang, and Z. Mao, "A hybrid genetic algorithm approach based on differential evolution for economic dispatch with valve-point effect," International Journal of Electrical Power \& Energy Systems, vol. 30, no. 1, pp. 31-38, 2008.

[6] B. Naama, H. Bouzeboudja, and A. Allali, "Solving the economic dispatch problem by using Tabu Search algorithm," in Proceedings of the TerraGreen International Conference on Advancements in Renewable Energy and Clean Environment, pp. 694-701, February 2013.

[7] M. A. Abido, "Multiobjective evolutionary algorithms for electric power dispatch problem," IEEE Transactions on Evolutionary Computation, vol. 10, no. 3, pp. 315-329, 2006.

[8] F. S. Wen and C. S. Chang, "Probabilistic approach for faultsection estimation in power systems based on a refined genetic algorithm," IEE Proceedings-Generation, Transmission and Distribution, vol. 144, no. 2, pp. 160-168, 1997.

[9] L. dos Santos Coelho and V. C. Mariani, "Particle swarm approach based on quantum mechanics and harmonic oscillator potential well for economic load dispatch with valve-point effects," Energy Conversion and Management, vol. 49, no. 11, pp. 3080-3085, 2008.

[10] J. S. Al-Sumait, J. K. Sykulski, and A. K. Al-Othman, "Solution of different types of economic load dispatch problems using a pattern search method," Electric Power Components and Systems, vol. 36, no. 3, pp. 250-265, 2008.

[11] L. D. S. Coelho and V. C. Mariani, "An improved harmony search algorithm for power economic load dispatch," Energy
Conversion and Management, vol. 50, no. 10, pp. 2522-2526, 2009.

[12] T. Yalcinoz, H. Altun, and M. Uzam, "Economic dispatch solution using a genetic algorithm based on arithmetic crossover," in Proceedings of the IEEE Porto Power Tech Conference (PPT '01), Porto, Portugal, September 2001.

[13] W.-M. Lin, F.-S. Cheng, and M.-T. Tsay, "An improved tabu search for economic dispatch with multiple minima," IEEE Transactions on Power Systems, vol. 17, no. 1, pp. 108-112, 2002.

[14] D. T. Phan and A. Koc, "Optimization approaches to securityconstrained unit commitment and economic dispatch with uncertainty analysis," in Optimization and Security Challenges in Smart Power Grids, Energy Systems, pp. 1-37, Springer, Berlin, Germany, 2013.

[15] Ü. B. Filik and M. Kurban, "Solving unit commitment problem using modified subgradient method combined with simulated annealing algorithm," Mathematical Problems in Engineering, vol. 2010, Article ID 295645, 15 pages, 2010.

[16] A. R. Balbo, M. A. da Silva Souza, E. C. Baptista, and L. Nepomuceno, "Predictor-corrector primal-dual interior point method for solving economic dispatch problems: a postoptimization analysis," Mathematical Problems in Engineering, vol. 2012, Article ID 376546, 26 pages, 2012.

[17] J. Li, D. Niu, and Y. Wu, "A parallel adaptive particle swarm optimization algorithm for economic/environmental power dispatch," Mathematical Problems in Engineering, vol. 2012, Article ID 271831, 14 pages, 2012.

[18] D. Barbagallo, E. di Nitto, D. J. Dubois, and R. Mirandola, "A bio-inspired algorithm for energy optimization in a selforganizing data center," in Self-Organizing Architectures, vol. 6090 of Lecture Notes in Computer Science, pp. 127-151, 2010.

[19] A. Pereira-Neto, C. Unsihuay, and O. R. Saavedra, "Efficient evolutionary strategy optimization procedure to solve the nonconvex economic dispatch problem with generator constraints," IEEE Proceeding-Generation, Transmission and Distribution, vol. 152, no. 5, pp. 653-660, 2005.

[20] T. A. A. Victoire and A. E. Jeyakumar, "Discussion of "particle swarm optimization to solving the economic dispatch considering the generator constraints'"' IEEE Transactions on Power Systems, vol. 19, no. 4, pp. 2121-2122, 2004.

[21] L. D. S. Coelho and C.-S. Lee, "Solving economic load dispatch problems in power systems using chaotic and Gaussian particle swarm optimization approaches," International Journal of Electrical Power and Energy Systems, vol. 30, no. 5, pp. 297-307, 2008.

[22] D. P. Kothari and J. S. Dhilon, Power System Optimization, PHI Learning, New Delhi, India, 2010.

[23] L. D. S. Coelho, R. C. Souza, and V. C. Mariani, "Improved differential evolution approach based on cultural algorithm and diversity measure applied to solve economic load dispatch problems," Mathematics and Computers in Simulation, vol. 79, no. 10, pp. 3136-3147, 2009.

[24] E. H. Aarts, J. H. Korst, and P. J. van Laarhoven, "A quantitative analysis of the simulated annealing algorithm: a case study for the traveling salesman problem," Journal of Statistical Physics, vol. 50, no. 1-2, pp. 187-206, 1988.

[25] S. Kirkpatrick, C. D. Gelatt, and M. P. Vecchi, "Optimization by simulated annealing," Science, vol. 220, no. 4598, pp. 671-680, 1983.

[26] V. Černý, "Thermodynamical approach to the traveling salesman problem: an efficient simulation algorithm," Journal of Optimization Theory and Applications, vol. 45, no. 1, pp. 41-51, 1985. 
[27] J. A. Khan, M. A. Z. Raja, and I. M. Qureshi, "Novel approach for a van der Pol oscillator in the continuous time domain," Chinese Physics Letters, vol. 28, no. 11, Article ID 110205, 2011.

[28] H.-D. Chiang, J.-C. Wang, O. Cockings, and H.-D. Shin, "Optimal capacitor placements in distribution systems. I: a new formulation and the overall problem," IEEE Transactions on Power Delivery, vol. 5, no. 2, pp. 634-642, 1990.

[29] R. Hooke and R. Jeeves, "Direct search solution of numerical and statistical problems," Journal of Association for Computing Machinery, vol. 8, pp. 212-229, 1961.

[30] E. D. Dolan, R. M. Lewis, and V. Torczon, "On the local convergence of pattern search," SIAM Journal on Optimization, vol. 14, no. 2, pp. 567-583, 2003.

[31] M. A. Raja, J. A. Khan, and I. M. Qureshi, "A new stochastic approach for solution of Riccati differential equation of fractional order," Annals of Mathematics and Artificial Intelligence, vol. 60, no. 3-4, pp. 229-250, 2010.

[32] T. R. Ramanan, M. Iqbal, and K. Umarali, "A particle swarm optimization approach for permutation flow shop scheduling problem," International Journal for Simulation and Multidisciplinary Design Optimization, vol. 5, article A20, 6 pages, 2014.

[33] A. M. Elaiw, A. M. Shehata, and M. A. Alghamdi, "A model predictive control approach to combined heat and power dynamic economic dispatch problem," Arabian Journal for Science and Engineering, vol. 39, no. 10, pp. 7117-7125, 2014.

[34] S. Thum and R. Schilling, "Optimization of hydraulic machinery bladings by multilevel CFD techniques," International Journal of Rotating Machinery, vol. 2, pp. 161-167, 2005.

[35] Z. A. Vale, C. Ramos, P. Faria et al., "Comparison between deterministic and meta-heuristic methods applied to ancillary services dispatch," in Trends in Applied Intelligent Systems, vol. 6096 of Lecture Notes in Computer Science, pp. 731-741, Springer, Berlin, Germany, 2010.

[36] A. Ş. Uyar, B. Türkay, and A. Keleş, "A novel differential evolution application to short-term electrical power generation scheduling," International Journal of Electrical Power and Energy Systems, vol. 33, no. 6, pp. 1236-1242, 2011.

[37] K. P. Wong and Y. W. Wong, "Genetic and genetic/simulatedannealing approaches to economic dispatch," IEE Proceedings: Generation, Transmission and Distribution, vol. 141, no. 5, pp. 507-513, 1994.

[38] C.-T. Su and C.-T. Lin, "New approach with a Hopfield modeling framework to economic dispatch," IEEE Transactions on Power Systems, vol. 15, no. 2, pp. 541-545, 2000. 

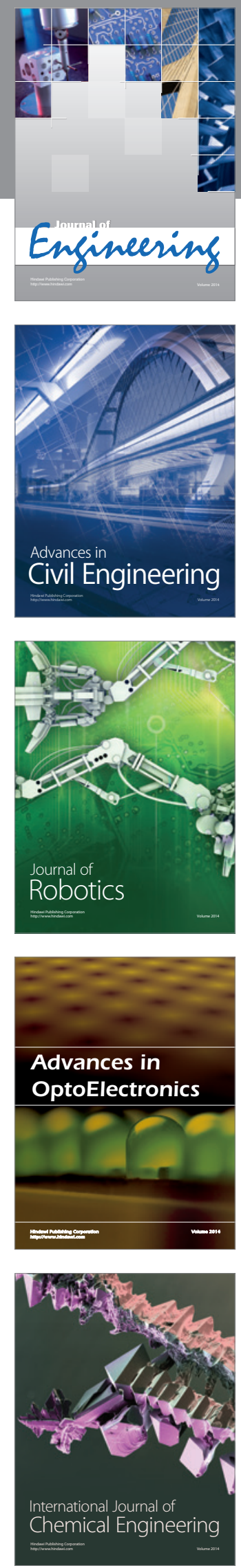

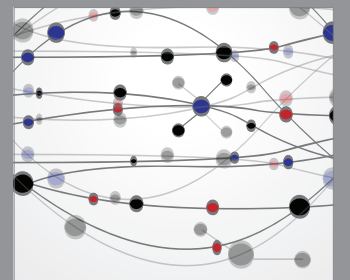

The Scientific World Journal
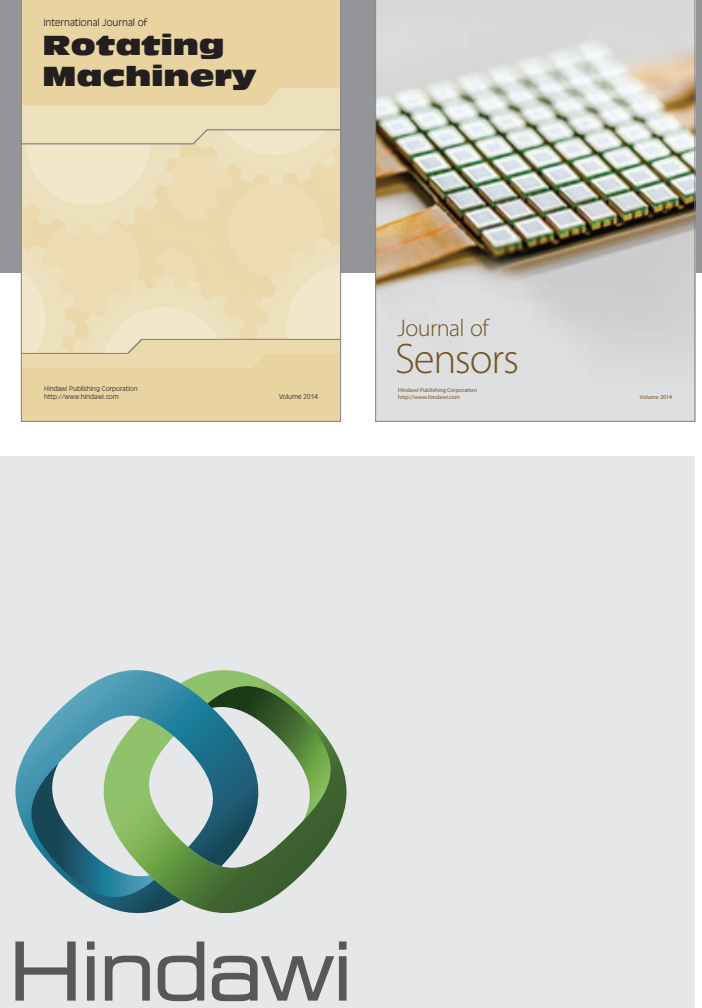

Submit your manuscripts at http://www.hindawi.com
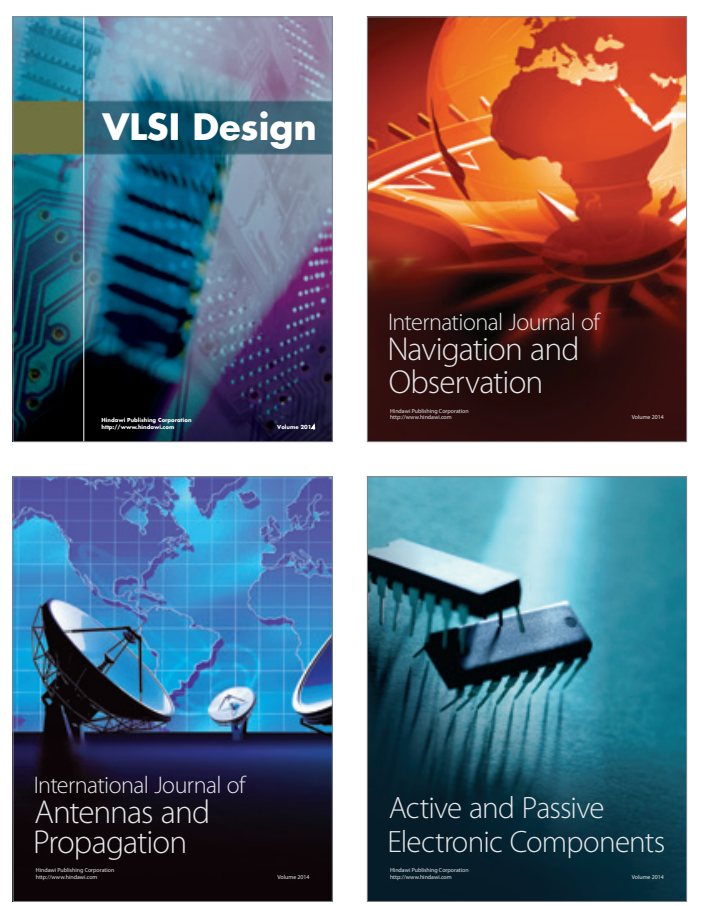
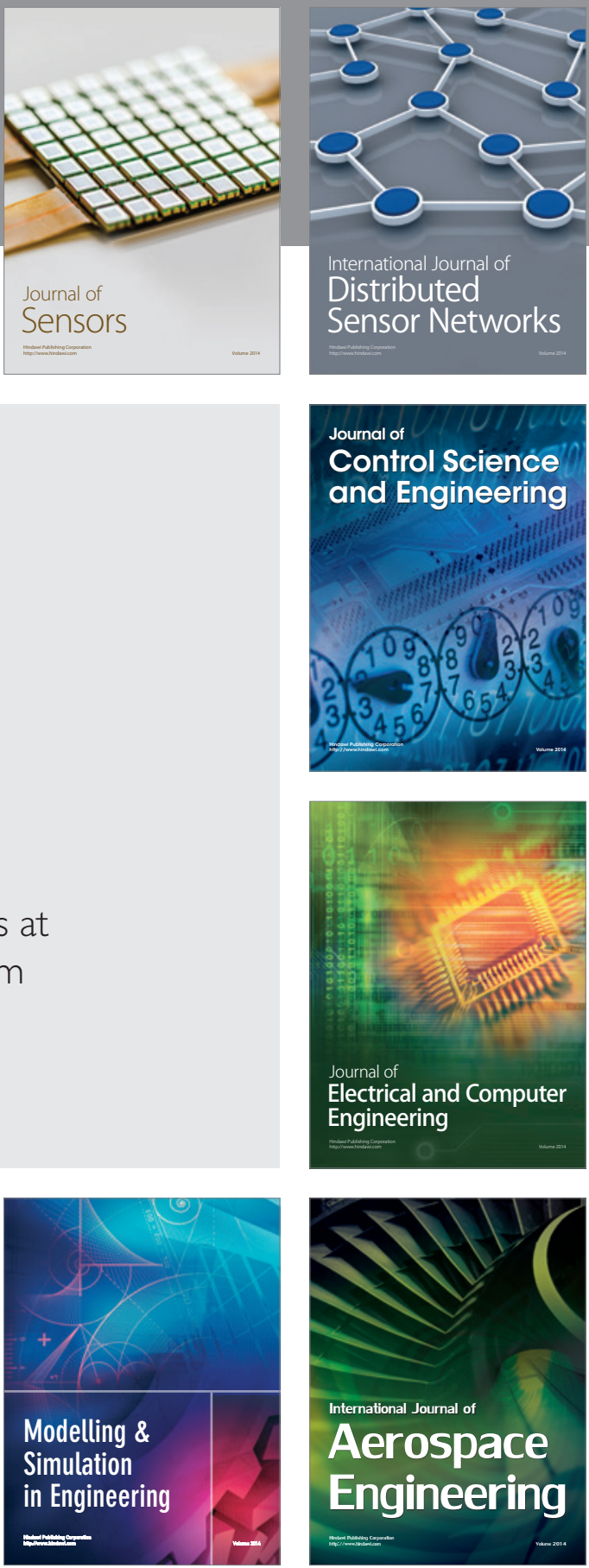

Journal of

Control Science

and Engineering
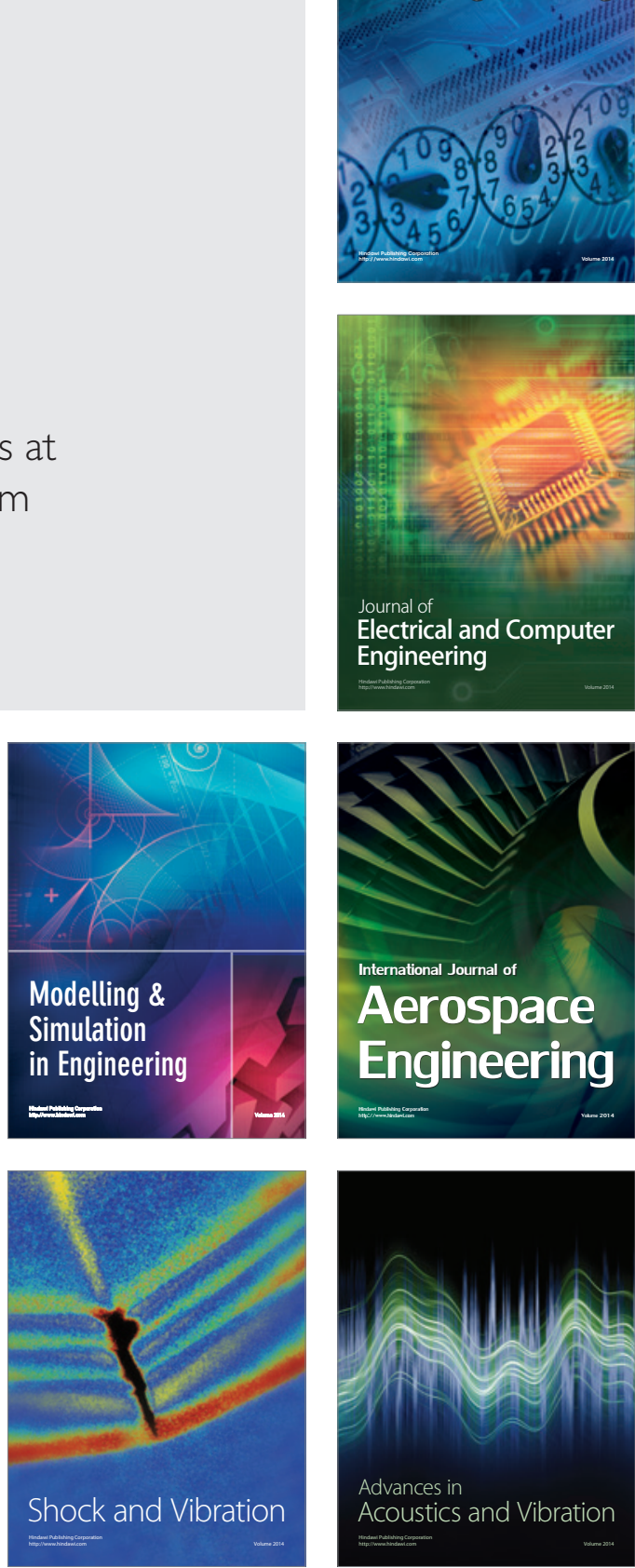